Research Article

\title{
A Quantitative Model to Calculate Gas Sealing Capacity and Design Sealing Parameters for Premium Connection
}

\author{
Honglin Xu $(\mathbb{D}$ and Bin Yang \\ School of Petroleum and Natural Gas Engineering, Chongqing University of Science and Technology, Chongqing 401331, China \\ Correspondence should be addressed to Honglin Xu; xuhlaca1986_jy@163.com
}

Received 29 December 2019; Accepted 3 February 2020; Published 24 February 2020

Academic Editor: Giovanni Lancioni

Copyright (c) 2020 Honglin Xu and Bin Yang. This is an open access article distributed under the Creative Commons Attribution License, which permits unrestricted use, distribution, and reproduction in any medium, provided the original work is properly cited.

\begin{abstract}
The present research studies about sealability of premium connection almost focus on qualitative description by sealing contact stress distribution, which is not convenient for sealing capacity evaluation and sealing parameter design. This paper proposes a quantitative model to calculate directly gas sealing capacity of cone to cone premium connection, different from the usual finite element method with testing, which combines the elastic thick wall cylinder interference fit theory for calculating sealing stress with the gas sealing criterion obtained from Murtagian's experimental results. With the proposed model, the effects of pipe wall thickness, seal cone taper, radial sealing interference, and axial sealing length on the gas sealing capacity have been investigated. Furthermore, the gas sealing capacity envelope curve based on radial sealing interference and axial sealing length is also calculated and a new sealing parameter design method is proposed for cone to cone premium connection. The results show that the internal upset pipe is good for sealability, and increasing both radial sealing interference and axial sealing length can significantly enhance gas sealability while seal cone taper has an unobvious effect on it. To meet sealing capacity, the designed sealing parameter combination $\left(\delta_{\mathrm{d}}, L_{\mathrm{d}}\right)$ should be located in the upper right region of the gas sealing capacity envelope curve.
\end{abstract}

\section{Introduction}

Tubing and casing premium connection usually adopts a proprietary metal-to-metal seal structure to meet a good gas sealability, and it has become one of the key technologies of maintaining wellbore integrity in harsh downhole conditions such as high pressure and high temperature (HPHT) gas wells, thermal recovery wells, and deepwater wells [1-3]. Metal-to-metal seal of premium connection produces sealing stress via radial interference fit, and a proper sealing stress distribution will directly affect its sealability. Consequently, how to evaluate the sealability and optimize the relevant sealing parameters appears to be of great importance for designing high-performance premium connection and guaranteeing the sealing integrity of tubing and casing strings.

The general sealing criterion for metal-to-metal seal is that the sealing contact stress should exceed the fluid pressure inside the string, which has also been taken as the early design criterion for tubing and casing connection [4]. However, this criterion has been established based on the assumption of complete smooth sealing surface and it does not conform to the actual rough contact and sealing. Gao et al. have analyzed the leakage resistance of rough contact in metal-to-metal seal and proposed increase of sealing contact stress and sealing length as larger as possible to enhance the sealability [5]. Murtagian et al. have established a gas sealing criterion from laboratory sealability tests for metal-to-metal seals, which adopts an integral of sealing stress multiplied by sealing length to represent the sealing capacity [6]. Xie et al. also proposed a similar sealability criterion for use in thermal well applications [7].

Based on those sealing criteria, sealability evaluation and sealing parameter design for premium connections have also been mainly focused on the sealing contact stress analysis by the theoretical model, field testing, and finite element method (FEM). Teodoriu and Badicioiu analyzed theoretically the sealing contact stress for the American Petroleum 
Institute (API) connection [8], and Xu et al. conducted the theoretical analysis of sphere to cone sealing performance for tubing and casing premium connection [9]. Hamilton et al. also adopted ultrasonic techniques to examine accurately the seal surface contact stress in premium connections [10]. Taken as an effective way to deal with the material nonlinearity, contact nonlinearity, and complex load conditions for premium connection, FEM has been the most popular and necessary tool for new product development of premium connection [11-14].

However, the present investigations are nearly qualitative methods, which are not convenient for sealing capacity evaluation and sealing parameter design. Consequently, this paper proposed a quantitative model to calculate directly gas sealing capacity of premium connection by combining the elastic thick wall cylinder interference fit theory for calculating sealing stress with the gas sealing criterion obtained from Murtagian's experimental results. Then, this paper also investigated the effects of pipe thickness, seal cone taper, radial sealing interference, and axial sealing length on the gas sealing capacity and further calculated the gas sealing capacity envelope curve of premium connection on the basis of radial sealing interference and axial sealing length and also proposed a new sealing parameter design method.

\section{Model Development for Gas Sealing Capacity of Premium Connection}

2.1. Radial Metal-to-Metal Seal in Premium Connection. Premium connection adopts radial metal-to-metal seal to satisfy high gas tight for tubing and casing strings. The metal-to-metal seal mainly includes two categories, namely, the conformal contact seal and nonconformal contact seal. The former refers to the typical cone to cone seal, and the latter generally includes the ball to cone seal, the ball to cylinder seal, and the ball to ball seal [1]. In this paper, the cone to cone seal structure is studied. Figures $1(\mathrm{a})$ and 1(b) show, respectively, the initial contact and final contact between pin end and coupling, which also clearly explain the radial interference development and gas sealability production of premium connection.

2.2. Sealing Contact Stress of Premium Connection. For calculating the gas sealing capacity of cone to cone premium connection, the sealing contact stress distribution should be made certain first. We simplified the contact between pin end and coupling into numerous tiny cylinder combinations; then, the sealing contact stress of premium connection can be calculated approximately by the elastic mechanics theory of thick wall cylinder interference fit. The radial interference fit between inner pin end and outer coupling is shown in Figure 2.

According to the elastic mechanics theory of thick wall cylinder interference fit [15], if a cylinder with an inner radius of $r_{\mathrm{i}}$ and outer radius of $r_{\mathrm{o}}$ is subjected to an inner pressure $p_{i}$ and an outer pressure $p_{0}$, the radial displacement at any radius in the cylinder can be expressed as

$$
u_{\mathrm{r}}=\frac{1-v}{E} \frac{r_{\mathrm{i}}^{2} p_{\mathrm{i}}-r_{\mathrm{o}}^{2} p_{\mathrm{o}}}{r_{\mathrm{o}}^{2}-r_{\mathrm{i}}^{2}} r+\frac{1+v}{E} \frac{r_{\mathrm{i}}^{2} r_{\mathrm{o}}^{2}\left(p_{\mathrm{i}}-p_{\mathrm{o}}\right)}{r_{\mathrm{o}}^{2}-r_{\mathrm{i}}^{2}} \frac{1}{r}
$$

For the inner pin cylinder in Figure 2, radial sealing contact stress $p_{c}$ can be regarded as outer pressure $p_{o}$ and inner pressure $p_{\mathrm{i}}$ is zero. Let $r_{\mathrm{i}}=r_{0}, r_{\mathrm{o}}=r=r_{\mathrm{c}}, p_{\mathrm{i}}=0$, and $p_{\mathrm{o}}=p_{\mathrm{c}}$ in equation (1), then the radial compressed displacement at the outer wall of the inner pin cylinder can be obtained as follows:

$$
\delta_{\text {pin }}=-\frac{r_{\mathrm{c}} p_{\mathrm{c}}}{E_{\text {pin }}}\left(\frac{r_{\mathrm{c}}^{2}+r_{0}^{2}}{r_{\mathrm{c}}^{2}-r_{0}^{2}}-\nu_{\text {pin }}\right) \text {. }
$$

For the outer coupling cylinder in Figure 2, radial sealing contact stress $p_{\mathrm{c}}$ can be regarded as inner pressure $p_{\mathrm{i}}$ and outer pressure $p_{\mathrm{o}}$ is zero. Let $r_{\mathrm{i}}=r=r_{\mathrm{c}}, r_{\mathrm{o}}=R_{0}, p_{\mathrm{i}}=p_{\mathrm{c}}$, and $p_{\mathrm{o}}=0$ in equation (1), then the radial stretched displacement at the inner wall of the coupling cylinder can be obtained as follows:

$$
\delta_{\text {cou }}=\frac{r_{\mathrm{c}} p_{\mathrm{c}}}{E_{\mathrm{cou}}}\left(\frac{R_{0}^{2}+r_{\mathrm{c}}^{2}}{R_{0}^{2}-r_{\mathrm{c}}^{2}}+v_{\mathrm{cou}}\right) .
$$

According to the radial deformation compatibility relation shown in Figure 2, the sum of $\delta_{\text {pin }}$ and $\delta_{\text {cou }}$ should be equal to the radial interference value $\delta$, so

$$
\left|\delta_{\text {pin }}\right|+\left|\delta_{\text {cou }}\right|=\delta \text {. }
$$

Now, substituting equations (2) and (3) into equation (4), the radial sealing contact stress $p_{c}$ can be expressed as

$$
p_{\mathrm{c}}=\frac{\delta}{r_{\mathrm{c}}\left[\left(1 / E_{\mathrm{pin}}\right)\left(\left(\left(r_{\mathrm{c}}^{2}+r_{0}^{2}\right) /\left(r_{\mathrm{c}}^{2}-r_{0}^{2}\right)\right)-\nu_{\text {pin }}\right)+\left(1 / E_{\text {cou }}\right)\left(\left(\left(R_{0}^{2}+r_{c}^{2}\right) /\left(R_{0}^{2}-r_{c}^{2}\right)\right)+\nu_{\text {cou }}\right)\right]} .
$$

Considering the variation of contact radius for the whole sealing contact surface and the fact that the normal contact stress contributes to gas sealability directly, then 


$$
p_{\mathrm{cN}}=\frac{\delta}{\left(r_{\mathrm{c} 0}-x \tan \gamma\right)\left[\left(1 / E_{\mathrm{pin}}\right)\left(\left(\left(r_{c}^{2}+r_{0}^{2}\right) /\left(r_{c}^{2}-r_{0}^{2}\right)\right)-v_{\mathrm{pin}}\right)+\left(1 / E_{\mathrm{cou}}\right)\left(\left(\left(R_{0}^{2}+r_{c}^{2}\right) /\left(R_{0}^{2}-r_{c}^{2}\right)\right)+v_{\mathrm{cou}}\right)\right] \cos \gamma} \quad(0 \leq x \leq L) .
$$

2.3. Gas Sealing Capacity of Premium Connection. In this paper, the gas sealing criterion from laboratory sealability tests for metal-to-metal seals has been chosen for gas sealability evaluation [6]. The integral of macro sealing stress multiplied by sealing length represents the sealing capacity of metal-to-metal seal, and it can be expressed as a gas sealing index $W_{\mathrm{a}}$. In HPHT gas wells,

$$
W_{\mathrm{a}}=\int_{0}^{L} p_{\mathrm{cN}}^{1.4}(x) \mathrm{d} x .
$$

Murtagian et al. also proposed that premium connection has reliable gas sealability only when $W_{\mathrm{a}}$ in equation (7) exceeds a critical value $W_{\text {ac }}$ :

$$
W_{\mathrm{ac}}=103.6 \times\left(\frac{p_{\mathrm{g}}}{p_{\mathrm{a}}}\right)^{0.838}\left(\mathrm{~mm} \cdot \mathrm{MPa}^{1.4}\right) .
$$

However, this evaluation criterion is too conservative because the gas leakage rate considered reliable for sealing in Murtagian's experiment is $0.025 \mathrm{~cm}^{3} / 15 \mathrm{~min}$, while the permitted limit gas leakage rate for casing and tubing connection is $0.9 \mathrm{~cm}^{3} / 15 \mathrm{~min}$ in ISO 13679 [16]. Considering this fact and the existing failure cases of tubing premium connection in HPHT gas wells, Xie further proposed a practical critical value of $W_{\mathrm{ac}}$ [17]:

$$
W_{\mathrm{ac}}=10 \times\left(\frac{p_{\mathrm{g}}}{p_{\mathrm{a}}}\right)^{0.838} .
$$

When the sealing contact stress distribution is known, by combining (7) and (9), the gas sealing capacity of premium connection in HPHT gas wells can be obtained as

$$
p_{\mathrm{gs}}=p_{\mathrm{a}}\left[\frac{1}{10} \int_{0}^{L} p_{\mathrm{cN}}^{1.4}(x) \mathrm{d} x\right]^{(1 / 0.838)} .
$$

\section{Sealing Parameter Sensitivity Analysis on Gas Sealing Capacity}

3.1. Basic Calculation Parameters. It can be seen from equation (10) that the gas sealing capacity of premium connection is closely related to sealing contact stress $p_{\mathrm{cN}}$, which is mainly decided by sealing parameters, material properties, and connection geometry parameters in equation (6). Because material properties and geometry parameters are usually constant for a special premium connection, sealing parameters turn out to be the key factors influencing its gas sealing capacity. Consequently, this paper takes a casing premium connection of P110 steel, $127 \mathrm{~mm}$ outer diameter, and $6.43 \mathrm{~mm}$ or $7.52 \mathrm{~mm}$ pipe wall thickness as an example to analyze the influences of the seal cone taper, radial sealing interference, and axial sealing length on the gas sealing capacity of premium connection. The basic calculated parameters are shown in Table 1.

3.2. Influence of Seal Cone Taper on Gas Sealing Capacity. Assuming that the radial sealing interference $\delta$ is $0.5 \mathrm{~mm}$ and the axial sealing length $L$ is $10 \mathrm{~mm}$, we have calculated the gas sealing capacity of cone to cone premium connection under the condition that seal cone taper $t$ is $1 / 5,1 / 10,1 / 20,1 /$ $30,1 / 40,1 / 50$, and $1 / 60$, respectively. The calculated results are shown in Figure 3.

It can be seen from Figure 3 that if $\delta$ and $L$ are both constant, gas sealing capacity increases gradually but more and more slowly with the decrease of seal cone taper and it is not nearly influenced by seal cone taper when it is smaller than $1 / 30$. Considering that gas sealability will be influenced more easier by axial working loads under a high value of seal cone taper, we suggest the practical seal cone taper value should be controlled with 1/30 1/10. Figure 3 also shows that the thicker the pipe wall, the better the gas sealability, which indicates that adoption of inner upset for pin end can enhance the gas sealing capacity of premium connection.

\subsection{Influence of Radial Sealing Interference on Gas Sealing} Capacity. Assuming that the seal cone taper $t$ is $1 / 20$ and the axial sealing length $L$ is $10 \mathrm{~mm}$, we have calculated the gas sealing capacity of cone to cone premium connection under the condition that radial sealing interference $\delta$ is $0.1 \sim 1 \mathrm{~mm}$. The calculated results are shown in Figure 4.

It can be seen from Figure 4 that if $t$ and $L$ are both constant, gas sealing capacity of premium connection increases a power law with the increase of radial sealing interference $\delta$. When the pipe wall thickness is $6.43 \mathrm{~mm}$ and radial sealing interference increases from $0.1 \mathrm{~mm}$ to $1 \mathrm{~mm}$, gas sealing capacity increases from $6.47 \mathrm{MPa}$ to $303.27 \mathrm{MPa}$ and by 46 times, which indicates that radial sealing interference will significantly influence gas sealability of cone to cone premium connection. For a needing sealed gas pressure, there exists a minimum value of radial sealing interference for meeting gas sealability. Taking a sealed gas pressure of $100 \mathrm{MPa}$ as an example, the radial sealing interferences should be at least larger than $0.51 \mathrm{~mm}$ and $0.39 \mathrm{~mm}$, respectively, for the two kinds of wall thickness connections.

3.4. Influence of Axial Sealing Length on Gas Sealing Capacity. Assuming that the seal cone taper $t$ is $1 / 20$ and the radial sealing interference $\delta$ is $0.5 \mathrm{~mm}$, we have also calculated the 


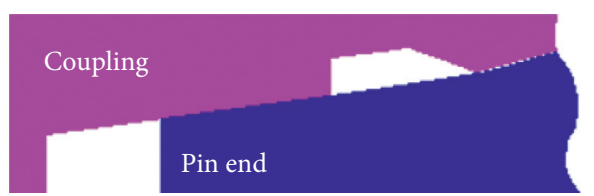

(a)

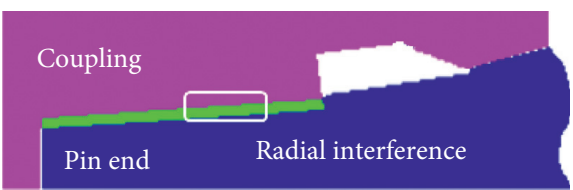

(b)

Figure 1: The schematic diagram of sealing contact for cone to cone premium connection. (a) Initial contact. (b) Final contact.

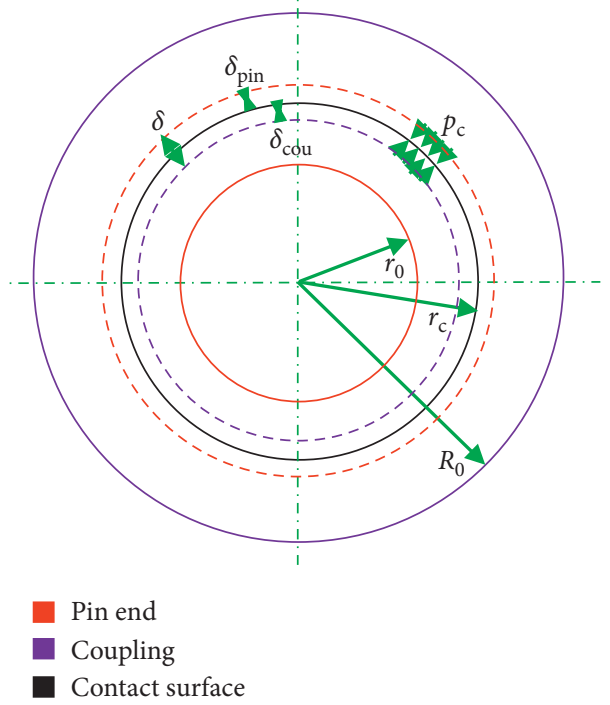

FIgURE 2: The schematic diagram of radial interference fit between inner pin end and outer coupling.

TABLE 1: The basic parameters for a casing premium connection.

\begin{tabular}{lccccccccc}
\hline$E_{\text {pin }}(\mathrm{MPa})$ & $E_{\text {cou }}(\mathrm{MPa})$ & $v_{\text {pin }}$ & $\nu_{\text {cou }}$ & $r_{\mathrm{c} 0}(\mathrm{~mm})$ & $R_{0}(\mathrm{~mm})$ & $p_{\mathrm{a}}(\mathrm{MPa})$ & $t$ & $\delta(\mathrm{mm})$ & $L(\mathrm{~mm})$ \\
\hline 206000 & 206000 & 0.28 & 0.28 & 59.77 & 70.65 & 0.101 & $1 / 20$ & 0.5 & 10 \\
\hline
\end{tabular}

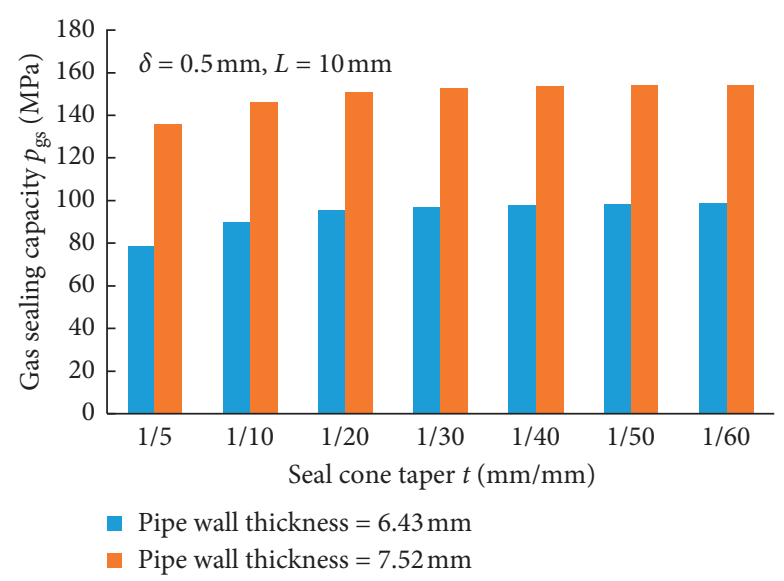

FIGURE 3: The effect of the seal cone taper on gas sealing capacity.

gas sealing capacity of cone to cone premium connection under the condition that axial sealing length $L$ is $1 \mathrm{~mm} 20 \mathrm{~mm}$. The calculated results are shown in Figure 5.
It can be seen from Figure 5 that if $t$ and $\delta$ are both constant, gas sealing capacity of premium connection increases linearly with the increase of axial sealing length. 


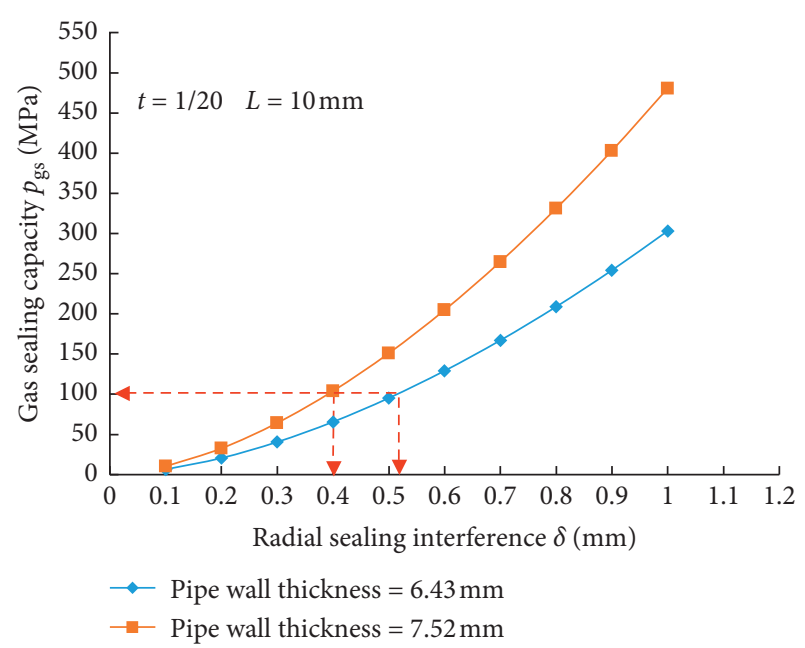

FIgURE 4: The effect of radial sealing interference on gas sealing capacity.

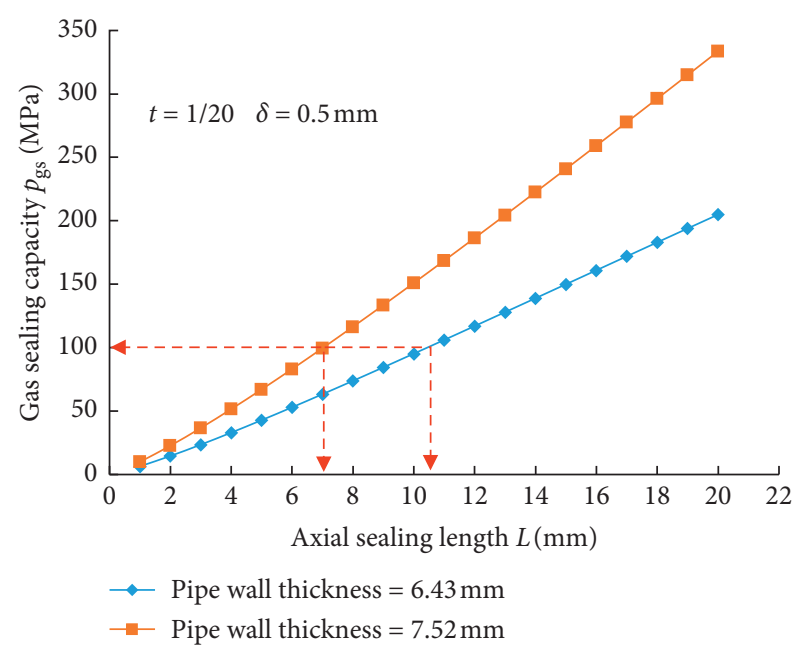

FIGURE 5: The effect of axial sealing length on gas sealing capacity.

When the pipe wall thickness is $6.43 \mathrm{~mm}$ and axial sealing length increases from $1 \mathrm{~mm}$ to $20 \mathrm{~mm}$, gas sealing capacity increases from $6.42 \mathrm{MPa}$ to $204.92 \mathrm{MPa}$ and nearly by 31 times, which indicates that axial sealing length will also significantly influence gas sealability of premium connection. For a needing sealed gas pressure, there also exists a minimum value of axial sealing length for meeting gas sealing capacity. Taking sealed gas pressure of $100 \mathrm{MPa}$ as an example, the axial sealing length $L$ should be at least larger than $10.44 \mathrm{~mm}$ and $7.02 \mathrm{~mm}$, respectively, for the two kinds of wall thickness connections.

\section{Sealing Parameter Design of Premium Connection}

It can be easily concluded from the analyzed results in Sections 3.2 3.4 that the main two parameters of effecting gas sealing capacity of cone to cone premium connection are radial sealing interference $\delta$ and axial sealing length $L$. In order to be convenient for optimizing these two main parameters, we have calculated the gas sealing capacity envelope curve between them under the condition that the pipe wall thickness is $6.43 \mathrm{~mm}$ and the needing sealed gas pressure are $70 \mathrm{MPa}$, $100 \mathrm{MPa}$, and $140 \mathrm{MPa}$, respectively. The calculated results are shown in Figure 6. The gas sealing capacity envelope curve means that the sealing parameter combination $(L, \delta)$ points on the curve can exactly seal the needing sealed gas pressure.

It can be seen from Figure 6 that if the needing sealed gas pressure and other conditions are constant, the needed radial sealing interference decreases gradually and the decrease rate is slower and slower with the increase of axial sealing length. To satisfy the gas sealing capacity, the designed sealing parameter combination $\left(L_{\mathrm{d}}, \delta_{\mathrm{d}}\right)$ should be located at the upper region of the envelope curve. That is to say, when the designed axial sealing length is shorter, we can select a relatively bigger radial sealing interference and when the designed axial sealing length is longer, we can select a relatively smaller radial sealing interference. However, considering the sealing surface may yield and the sealability also easily influenced by axial working loads under the former situation, $\left(L_{\mathrm{d}}, \delta_{\mathrm{d}}\right)$ should be properly located at the upper right region. For example, if the sealed gas pressure is $100 \mathrm{MPa}$ and the designed axial sealing length is $10 \mathrm{~mm}$, the designed radial sealing interference should be larger than $0.52 \mathrm{~mm}$. In a word, the gas sealing capacity envelope curve can be used for sealing parameter design easily.

\section{Conclusions and Future Work}

(1) By combining theoretical sealing stress and experimental gas sealing criterion, a quantitative model to calculate directly gas sealing capacity of cone to cone premium connection has been proposed.

(2) The effects of main sealing parameters on gas sealing capacity of cone to cone premium connection have been analyzed and show that the internal upset pipe is good for sealability and increasing both radial sealing interference and axial sealing length can significantly enhance gas sealability while seal cone taper has an unobvious effect.

(3) By calculating the gas sealing capacity envelope curve between axial sealing length and radial sealing interference, a new sealing parameter design method for cone to cone premium connection has been proposed, and the recommended sealing parameter combinations $\left(L_{\mathrm{d}}, \delta_{\mathrm{d}}\right)$ should be located at the upper right region of the envelope curve.

(4) This paper aims to propose a different method for sealability evaluation and sealing parameter design for premium connection, and the critical importance is how to get more accurate sealing contact stress distribution. Consequently, the sealing contact stress model, considering elasticplastic deformation of material, more complex downhole loads, and sealing structure type, should be further investigated to perfect the proposed method. 


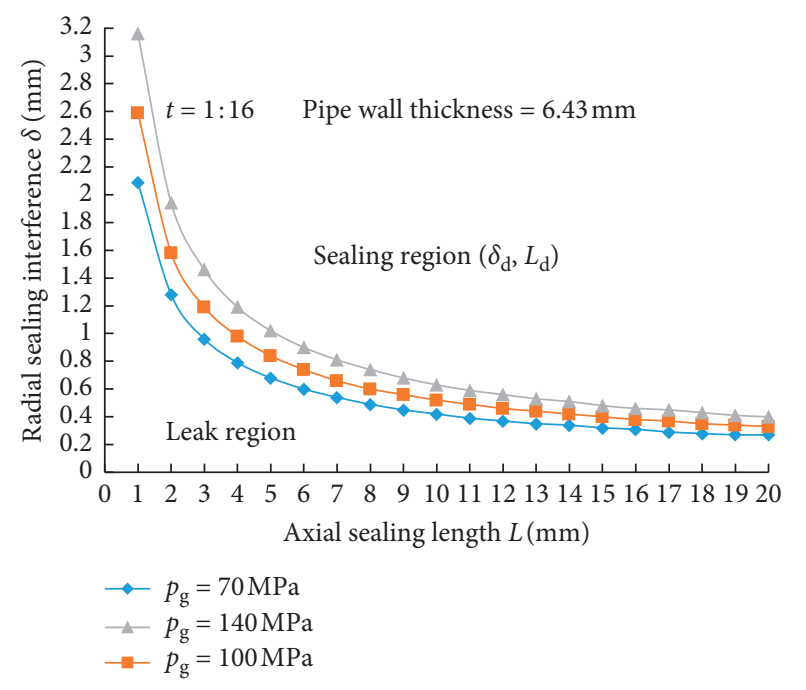

FIgURE 6: The gas sealing capacity envelope curve of cone to cone premium connection.

\section{Nomenclature}

$E, E_{\text {pin }}$, and Elasticity modulus of cylinder, pin end, and $E_{\text {cou }}$ ～coupling, respectively, $\mathrm{MPa}$

L: $\quad$ Axial length of cone to cone sealing structure, $\mathrm{mm}$

$L_{\mathrm{d}}$ : The designed axial length of cone to cone sealing structure, $\mathrm{mm}$

$p_{\mathrm{a}}: \quad$ Atmospheric pressure, $\mathrm{MPa}$

$p_{\mathrm{g}}$ : $\quad$ Gas pressure needed to be sealed, $\mathrm{MPa}$

ps: Gas sealing capacity of premium connection, $\mathrm{MPa}$

$p_{\mathrm{i}}$ and $p_{\mathrm{o}}$ : Inner and outer pressure acted on the thick wall cylinder, respectively, MPa

$p_{\mathrm{c}}$ : $\quad$ Radial sealing contact stress resulted from radial interference, $\mathrm{MPa}$

$p_{\mathrm{cN}}$ : Normal sealing contact stress resulted from radial interference, $\mathrm{MPa}$

$r_{\mathrm{c}}$ : $\quad$ Contact radius between inner pin end and outer coupling, $\mathrm{mm}$

$r_{0}$ : Radius at inner wall of pin end, $\mathrm{mm}$

$r_{\mathrm{c} 0}$ : Sealing radius at the larger end of cone to cone sealing structure, $\mathrm{mm}$

$R_{0}$ : $\quad$ Radius at outer wall of coupling, $\mathrm{mm}$

$r, r_{\mathrm{i}}$, and $r_{\mathrm{o}}$ : Radius at any point, inner wall, and outer wall of thick-walled cylinder, respectively, $\mathrm{mm}$

$u_{r}: \quad$ Radial displacement at any radius of thickwalled cylinder, $\mathrm{mm}$

$W_{\mathrm{a}}$ : Gas sealing index for metal-to-metal seal, $\mathrm{mm} \cdot \mathrm{MPa}^{1.4}$

$W_{\text {ac }}: \quad$ Critical gas sealing index, $\mathrm{mm} \cdot \mathrm{MPa}^{1.4}$

$\delta_{\text {cou }}$ : Radial stretched displacement at the inner wall of the outer coupling cylinder, $\mathrm{mm}$

$\delta_{\text {pin }}: \quad$ Radial compressed displacement at the outer wall of the inner pin cylinder, $\mathrm{mm}$

$\delta$ : $\quad$ Radial interference value between inner pin end and outer coupling, $\mathrm{mm}$ $\delta_{\mathrm{d}}: \quad$ The designed radial interference value between inner pin end and outer coupling, $\mathrm{mm}$

$\gamma: \quad$ Cone angle, can be calculated by seal cone taper $t$ as $\tan \gamma=0.5 t$, rad

$\nu, v_{\text {pin }}$, and Poisson's ratio of cylinder, pin end, and

$\nu_{\text {cou }}:$ coupling, respectively, dimensionless.

\section{Data Availability}

The data used to support the findings of this study are available from the corresponding author upon request.

\section{Conflicts of Interest}

The authors declare that they do not have any commercial or associative interest that represents a conflicts of interest in connection with the work submitted.

\section{Acknowledgments}

This research was funded by the National Natural Science Foundation of China (Grant no. 51804060), the Basic and Frontier Research Programs of Chongqing Science and Technology Commission of China (Grant no. cstc2018jcyjAX0614), the Science and Technology Research Program of Chongqing Municipal Education Commission (Grant no. KJQN201901519), and the Research Foundation of Chongqing University of Science and Technology (Grant no. CK2016B13).

\section{References}

[1] A. Bradley, S. Nagasaku, and E. Verger, "Premium connection design testing and installation for HPHT sour wells," in Proceedings of the SPE High Pressure/High Temperature Sour Well Design Applied Technology Workshop, May 2005.

[2] G. Carcagno, "The design of tubing and casing premium connections for HTHP wells," in Proceedings of the SPE High Pressure/High Temperature Sour Well Design Applied Technology Workshop, May 2005.

[3] W. Bassarath, M. Lafuente, and R. Branly, "Development and qualification of a next generation gas tight connection incorporating metal-to-metal Technology," in Proceedings of the International Petroleum Technology Conference, December 2015.

[4] API, "Bulletin on formulas and calculations for casing, tubing, drill pipe and line pipe properties," API Bulletin, vol. 5C3, 1994.

[5] L. Gao, "Seal design of premium threaded casing connections," Chinese Journal of Mechanical Engineering, vol. 41, no. 3, pp. 216-220, 2005.

[6] G. R. Murtagian, V. Fanelli, J. A. Villasante, D. H. Johnson, and H. A. Ernst, "Sealability of stationary metal-to-metal seals," Journal of Tribology, vol. 126, no. 3, pp. 591-596, 2004.

[7] J. Xie, C. Matthews, and A. Hamilton, "A study of sealability evaluation criteria for casing connections in thermal wells," in Proceedings of the SPE Canada Heavy Oil Technical Conference, June 2016.

[8] C. Teodoriu and M. Badicioiu, "Sealing capacity of API connections-theoretical and experimental results," SPE Drilling \& Completion, vol. 24, no. 1, pp. 96-103, 2009. 
[9] H. L. Xu, T. L. Li, B. Yang et al., "Theoretical analysis of sphere to cone sealing performance for tubing and casing premium connection," Journal of Southwest Petroleum University (Science \& Technology Edition), vol. 38, no. 5, pp. 179-184, 2016.

[10] K. A. Hamilton, B. Wagg, and T. Roth, "Using ultrasonic techniques to accurately examine seal-surface-contact stress in premium connections," SPE Drilling \& Completion, vol. 24, no. 4, pp. 696-704, 2009.

[11] L. Hilbert Jr. and I. Kalil, "Evaluation of premium threaded connections using finite-element analysis and full-scale testing," in Proceedings of the SPE/IADC Drilling Conference, February 1992.

[12] J. Takano, M. Yamaguchi, and H. Kunishige, "Development of premium connection "KSBEAR" for withstanding high compression, high external pressure, and severe bending," vol. 47, Kawasaki Steel Technical Report-English edition, pp. 14-22, JFE Steel Corporation, Tokyo, Japan, 2002.

[13] M. Zhang, Q. Zhang, L. Cheng et al., "Study on the performances of two kinds of special tubing connector," Chemical Engineering Transactions, vol. 46, pp. 1063-1068, 2015.

[14] W. Chen, Q. Di, H. Zhang, F. Chen, and W. Wang, "The sealing mechanism of tubing and casing premium threaded connections under complex loads," Journal of Petroleum Science and Engineering, vol. 171, pp. 724-730, 2018.

[15] Z. L. Xu, Elasticity, Higher Education Press, Beijing, China, 2006, in Chinese.

[16] ISO, "Petroleum and natural gas industries-Procedures for testing casing and tubing connections," ISO, Geneva, Switzerland, 1st edition, 2002.

[17] J. Xie, "Numerical evaluation of tubular connections for HPHT applications," in Proceedings of the Baosteel BAC, Shanghai, China, June 2013. 